
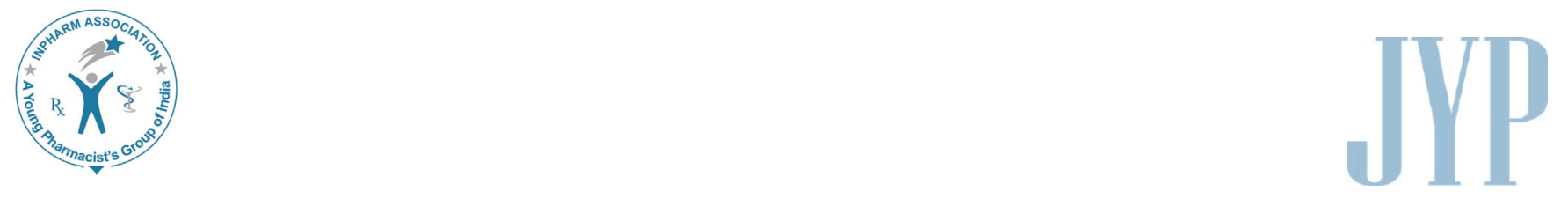

\title{
Assessing the Magnitude and Effect of Various Risk Factors Associated with Vitamin D Deficiency among Females in the UAE
}

\author{
Muaed Jamal Alomar* and Marie Christine Al Khouri \\ College of Pharmacy and Health Sciences, Alain University of Science and Technology, Alain, UAE.
}

\begin{abstract}
Aim: The aim of this research is to determine the incidence and risk of developing Vitamin $D$ deficiency, specifically among females within a certain age range in the UAE. Materials and Methods: A cross-sectional, descriptive, and observational study to observe and record lifestyle and dietary risk factors and their frequency in the selected females' daily lives and routines. An online distributed questionnaire has been designed and distributed among women of Al Ain city in Abu Dhabi (UAE). Statistical Analysis: SPSS version 20 was used for data analysis and statistical testing. The significance was tested using Chi Square. Results: Among the 124 females, 35.5\% were exposed to the sun between 10 am and $3 \mathrm{pm}$ and $64.5 \%$ were not exposed. Vit D rich food consumption includes: Cod liver oil ( $10 \%$ Yes, $90 \%$ No), Salmon ( $26.6 \%$ Yes, $73.4 \%$ No), Tuna (44.4\% Yes, 55.6\% No), Sardines (21.8\% Yes, $78.2 \%$ No), Liver/Beef (33.1\% Yes, 66.9\% No), Orange Juice fortified ( $50 \%$ Yes, $50 \%$ No), Milk fortified $(41.9 \%$ Yes, $58.1 \%$ No), Yoghurt fortified ( $46 \%$ Yes, $54 \%$ No), and Cereal fortified $(29.8 \%$ Yes, $70.2 \%$ No). A high degree of association with P-values of 0.007 Pearson Chi-Square for frequent use of Sun protection factor 15 and its effect on sun exposure being daily in an in doors work/education environment, and Pvalues of 0.046 and 0.028 corresponding to "usual" use of Sun protection factor 15 and "rare" use. Conclusion: Vitamin D deficiency is common among female women who live in the UAE; many factors contribute to this deficiency. More rigorous work is needed to encourage women to avoid some of these factors in order to promote health and prevent vitamin $D$ deficiency related health problems.
\end{abstract}

Key words: Bone, Calcium, Sun protection factor, Sun, Vitamin D.

\section{INTRODUCTION}

Vitamin D has long been considered as an important factor affecting the health of the human body. Numerous studies have been conducted suggesting the role of Vitamin D in

\begin{tabular}{|c|l|}
\hline \multicolumn{2}{|c|}{ Access this article online } \\
\hline \multirow{2}{*}{ Journal Sponsor } & $\begin{array}{l}\text { Website: } \\
\text { www.jyoungpharm.org }\end{array}$ \\
\cline { 2 - 2 } & $\begin{array}{l}\text { DOI: } \\
\text { www.phcog net }\end{array}$ \\
& \\
\cline { 2 - 2 } & \\
\hline
\end{tabular}

the human body and therefore its significance and the effect of different factors on Vitamin D deficiency inclusive of: lifestyle-related factors and habits, age, gender, geographical locations, genetic factors, etc. Further studies have analyzed the genetic determinants of Vitamin D deficiency and its endogenous production in the body. The photosynthesis of Vitamin D has been occurring from an evolutionary perspective for more than 750 million years, where early vertebrates used up scarce sources of Vitamin D before venturing inwards from sea to land in order to preserve their skeletons as a result of rigid calcification ${ }^{1}$ Due to extensive

*Address for correspondence:

Dr. Muaed Jamal Alomar, PhD (Clinical Pharmacoinformatics), Lecturer, Clinical Pharmacy Department, College of Pharmacy and Health Sciences, Alain University of Science and Technology, Alain, UAE. E-mail: muayyad74@yahoo.com 


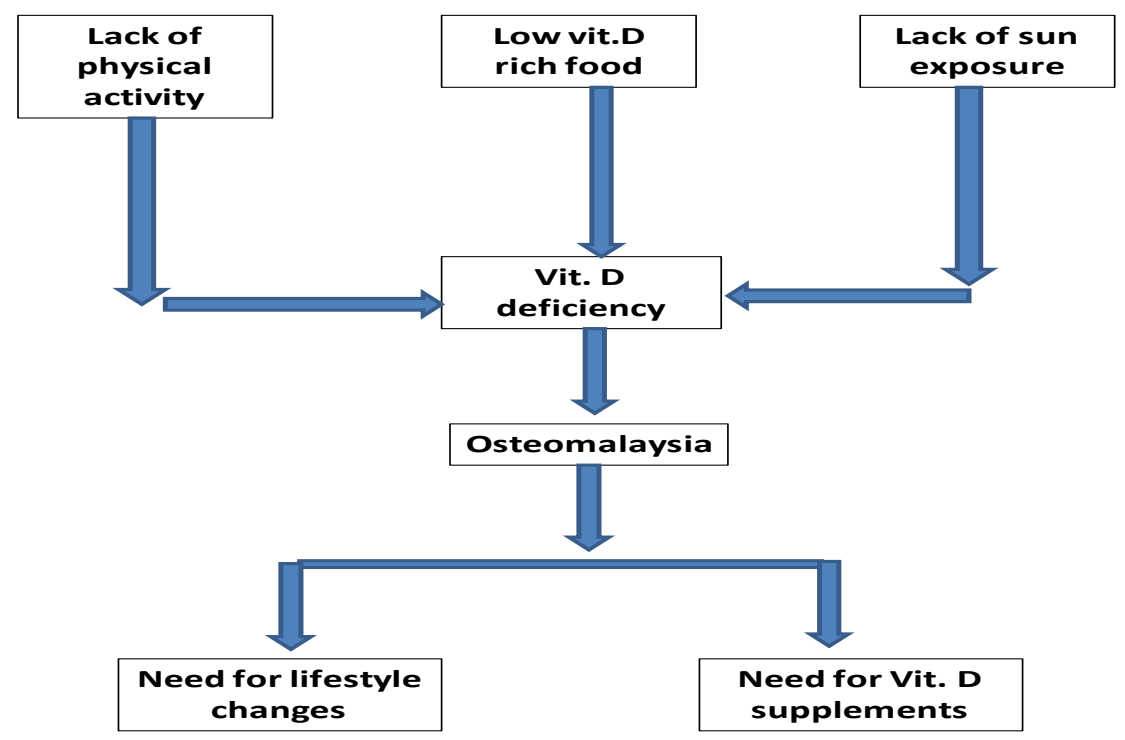

Graphical Abstract

migration, urbanization, and leading a modern industrialized lifestyle caused decreased sun exposure in the long term ${ }^{2}$ Glissen, DeBoot, and Whistler recognized that children living in the inner cities throughout Europe demonstrated growth retardation and developed skeletal deformities ${ }^{3}$ Further conclusions were reached in 1919 by Huldschinsky and in 1921 by Steenbock and Black in relation to other factors affecting Vitamin D deficiency and prevention, including exposure of children with rickets to a mercury arc lamp and the inclusion of fortified milk and other foods with synthetic Vitamin $\mathrm{D}_{2}{ }_{2}^{4}$ However, after inadequate and excess fortification of Vitamin D in milk products after World War II, an outbreak of intoxication occurred which led to banning of the fortification process of dairy products in most European countries until the present day ${ }^{5}$ Therefore, factors leading to the increase of Vitamin D deficiency and related harmful diseases having occurred decades ago proves the necessity of awareness, even in the current day, with further knowledge of deficiency-causing determinants and methods of prevention.

The physiology of Vitamin D, its endogenous photosynthesis, as well as exogenous properties are important in order to determine the comparative association between the risk factors associated with Vitamin D deficiency and the degree to which each factor contributes to the risk of its development. Vitamin D is a fat-soluble vitamin present naturally in few foods, added through fortification in several dietary foods, and as synthetically manufactured supplements. Being a hormone precursor, its natural presence and in fortified foods is of the Ergocalciferol form, or vitamin $\mathrm{D}_{2}$, whereas endogenously synthesized in the skin by sunlight is of the Cholecalciferol form (vitamin
$\left.\mathrm{D}_{3}\right)^{6}$ "Vitamin D controls calcium absorption in the small intestine and works with parathyroid hormone to mediate skeletal mineralization and maintain calcium homeostasis in the blood stream" "Along with calcium absorption, Vitamin $\mathrm{D}$ is obtained exogenously must be activated in the body in order to be metabolized by the liver and the kidneys.

The aim of this research study is to determine the incidence and risk of developing Vitamin D deficiency, specifically among females within a certain age range in the UAE, according to various well-known risk factors including: sun exposure and its determinants, dietary and fortified intake of Vitamin D, as well as obesity and BMI.

\section{MATERIALS AND METHODS}

A cross-sectional, descriptive, and observational study to observe and record lifestyle and dietary risk factors and their frequency in the selected females' daily lives and routines was used. The current risk of developing Vitamin $\mathrm{D}$ deficiency and its relation to these factors is analyzed in order to result in a sound conclusion of which factor(s) contribute(s) most to risk of developing deficiency.

The inclusion criteria are female, age 18-45. The exclusion criteria are male gender, below 18 or above 45 , pregnant and breastfeeding women including women already taking Vitamin D supplements during pregnancy, subjects that are known to be highly exposed to the sun on a daily and frequent basis in relation to their nature of work or residence (e.g. workers that are exposed to heat and sun daily), specific geographic areas in which sun exposure is either limited or high. Any other confounding factors that 
might externally affect the results, including but not limited to: genetic factors, nationality, skin pigmentation, patients undergoing strict treatment with supply of Vitamin D, etc.

The target population of this study represents a random sample of 124 females, aged 18 years to 45 years, from the United Arab Emirates. The duration of the study is approximately four months. A questionnaire in both English and Arabic languages comprising of 11 closeended questions regarding lifestyle and dietary routine and habits relating to factors affecting sun exposure and dietary intake of Vitamin D was distributed to a random sample of females across two cities of the UAE, $\mathrm{Al}$ Ain and Abu Dhabi Emirate (the Capital). The subjects were aware, prior to filling out the questionnaire, of being required not to answer should they be currently pregnant/breast feeding, or not within the inclusion age range mentioned above. The questionnaires were distributed online at the following source in the English language version: https://mcalk. typeform.com/to/wJYFm3, and at the following source in the Arabic language version: https:/ /apps.facebook.com/ my-surveys/qeeneh.

All statistical analyses were conducted using the IBM SPSS Statistics program version 20 . The statistics calculated using SPSS comprised frequencies of responses to the categories of each variable (risk factor) and cross-tabulation of interrelated factors. The variables (examined risk factors) represented nominal (categorical) and ordinal (categorical in an order) data, therefore SPSS descriptive cross-tab analysis was used, specifically using the Chi-Square test in order to determine significant association between two or more variables from the columns and rows.

\section{RESULTS}

The distributed questionnaire comprised of 11 variables. The variables required descriptive nominal and ordinal possible answer choices in order to facilitate the response method of individuals, due to the study relying on personal response and opinion rather than numerical data or accurate serum

\begin{tabular}{|cc|}
$\begin{array}{c}\text { Table 1: Food consumption among participants } \\
\text { Type of Vit. D rich food }\end{array}$ & $\begin{array}{c}\text { Consumption rate } \\
\text { Cod liver oil }\end{array}$ \\
\hline Tuna & $10 \%$ \\
\hline Salmon & $44 \%$ \\
\hline Sardine & $26 \%$ \\
\hline Liverorbeef & $22 \%$ \\
\hline Orangejuice & $33 \%$ \\
\hline Cereal & $50 \%$ \\
\hline Milk & $30 \%$ \\
\hline Yoghurt & $42 \%$ \\
\hline
\end{tabular}

analyses. The variables represented and mentioned in the objectives above are, respectively: sun exposure (or not) during a specific timing (between 10 am and 3 pm), type of Sun protection factor $(\mathrm{SPF})(8,15$, more than 15 , none), frequency of Sun protection factor application (frequently, usually/sometimes, rarely), frequency of practicing sunbathing (frequently, sometimes/during holidays, rarely), type of clothes and coverage (covering the whole body with religious "Hijab", not covering the whole body with arms, face, or legs uncovered), daily work/educational environment (indoors or a mix of outdoors and indoors according to the type of daily transportation used), nature of physical activity usually practiced (active lifestyle, usually practicing outdoor activities, sedentary lifestyle), types of food containing natural and fortified Vitamin D and choice of more than one type frequently consumed (cod liver oil, salmon, tuna, sardines, liver/beef, orange juice, milk, yoghurt, and readymade cereals), and finally current weight range (underweight, normal weight, overweight, obese).

The United Arab Emirate (UAE) is located in the Arabian Desert with plenty of sunshine. It has been assumed that people will get a sufficient amount of Vit. D from the sun. In this study 124 women participated in a survey about factors affecting Vit. D concentration, $65 \%$ of them have no exposure to sun light between 10 A.M to 3 P.M, 50\% rarely go sunbathing, $74 \%$ use sunscreen before they go outside, $44 \%$ wear hijab (Muslim dress code), 62\% have no sun exposure during their working hours and 38\% have a little exposure to sunlight during work. Table 1 illustrates food habits among all participants. Even though 61\% show normal body weight, only $40 \%$ practice occasional outdoor physical activity, see Table 1 for more details.

Twenty percent $(20 \%)$ of the participants have sedentary lifestyle and work indoor with no daily sun exposure, $55 \%$ have no sun exposure between 10 A.M-3 P.M and no consumption of cod liver oil, $31 \%$ get some exposure to sun light but they use sun screens.

A cross-tabulation represents interrelation between the following factors: sun exposure (between 10am-3pm or not), type of clothes (covered or not), and frequency of sunbathing (frequent, sometimes/during the holidays, rarely). Twenty-one women have the following three criteria according to the study: they are not exposed to the sun between 10 A.M. and 3 P.M., they rarely sunbathe and they cover their whole bodies which may indicate a vitamin $\mathrm{D}$ deficiency.

Another cross-tabulation interrelated the following factors/ determinants: the constant sun exposure (between 10 
am-3 pm or not) against which other factors' effects are observed, cross-tabulated with frequency of sunscreen application, type of sunscreen (Sun protection factor value), and most importantly nature of work/educational environment (indoors only or a mix of outdoors and indoors). The results depict the most significant association in comparison to the other factors; females frequently using Sun protection factor 8 (previously stated to reduce Vitamin $\mathrm{D}_{3}$ synthesis by $>95 \%$ ) are daily in a mix of outdoors and indoors work/educational environments depending on their methods of transport or nature of work, therefore a larger number, 4 , exposed to the sun than those that aren't exposed ( 1 female). This clearly indicates females' need to protect them from the exposure to the sun daily knowing their nature of work/ educational environment requires some exposure to the sun. However, their frequent use of Sun protection factor 8 will negatively impact Vitamin $\mathrm{D}_{3}$ synthesis in the skin, thus even though their work environment allows for some degree of sun exposure their frequent use of sunscreen will contribute further to development of Vitamin D deficiency. One can conclude initially for the females' work/ education environment affecting their choice of using sunscreen, therefore leading to inadequate sun exposure and reduced synthesis of Vitamin $\mathrm{D}_{3}$. Moreover, as the initial difference in frequencies between females' work/ education environment being "outdoors and indoors" or "only indoors" was visibly large, where "indoors only" is prevalent, the cross-tabulation results confirms the issue of a high percentage of females in the UAE working/learning indoors in buildings equipped with air conditioning. For example, females frequently using Sun protection factor 15, which reduces Vitamin $\mathrm{D}_{3}$ synthesis by $>98 \%$, as well as being daily indoors in their work/education environments thus being deprived of sun exposure, are 13 females compared to 2 being in a mix of indoors and outdoors work/education environments. Therefore, the cumulative effects of frequent use of Sun protection factor 15, being in work/education environments that are enclosed, with no exposure to the sun leads to a higher risk of developing Vitamin D deficiency. Even females rarely applying Sun protection factor in general, regardless of the type, are higher in number and in daily indoors work/education environment as well as not being exposed to the sun, 14 females, in comparison to 6 of the same category however exposed to the sun. A similar conclusion regarding the significance of the work/education environment can be stated, where it affects the interrelation between the other factors and undermines the choice of Sun protection factor value and frequency of application, as well as defining the extent of sun exposure. Being the most important factor, Chi-square tests depict a significant association of this factor to others with P- values of 0.007 Pearson ChiSquare for frequent use of Sun protection factor 15 and its effect on sun exposure being daily in an indoor work/ education environment, and P-values of 0.046 and 0.028 corresponding to "usual" use of Sun protection factor 15 and "rare" use (under similar circumstances above).

\section{DISCUSION}

According to this study, a preliminary inference in relation to the most obviously significant and meaningful determinants of Vitamin D intake, thus the most possible risk factors that can cumulatively lead to an increased risk of developing Vitamin D deficiency. Therefore, as clearly seen from the above frequencies, the most striking results are represented by the following variables: exposure or not to direct sunlight between 10am and $3 \mathrm{pm}$, frequency of sunbathing, type of sunscreen and frequency of application, and most importantly work/ education environment. The determinant/variable of sun exposure is considered to be the constant on which other factors depend, therefore cross-tabulation descriptive statistics in SPSS was performed interrelated with the sun exposure variable in order to conclude the effect of other determinants on the females' exposure to the sun or not.

Vitamin D deficiency should be considered as a very important factor for one's well-being, not only for prevention of classic rickets among children or osteomalacia among adults and the elderly, but also due to "demonstrated increase risks of many common cancers, type 1 diabetes, rheumatoid arthritis, and multiple sclerosis, and there are indications that they may be associated with type 2 diabetes and schizophrenia" 8 Vitamin D deficiencies can occur due to less than recommended levels of dietary intake over time, limited exposure to sunlight, the kidneys not being able to convert it to the active form, or inadequate absorption of vitamin $\mathrm{D}$ from the gastrointestinal tract ${ }^{9}$ According to a study on Vitamin D deficiency among University students in Abu Dhabi, deficiency was only recently found to be prevalent in the Gulf Region despite the fact of having a sunny climate and being at favorable latitude, both of which are major factors contributing to the adequate possible exposure to Vitamin $\mathrm{D}^{10}$ Also, studies have shown Emirati women to suffer from osteoporosis as a result of mild to severe Vitamin D deficiency as well as having low Vitamin D serum concentrations due to limited sun exposure and low dietary intake, further explains the prevalence of Vitamin D deficiency in the Middle East and the risk factors affecting its development despite the abundance of sunshine, thus registering the highest rates of rickets 
worldwide ${ }^{11}$ The common factors are: limited sun exposure due to cultural practices and behavior and prolonged breast-feeding without the intake of supplements.

International study conducted in women with osteoporosis, the highest proportion of hypovitaminosis $\mathrm{D}$ was noted in the Middle East. Inadequate vitamin D intake, urban dwelling, female gender, winter season, age, and high parity were independent predictors of low vitamin D levels. ${ }^{12}$

Lifestyle-related factors that alter the endogenous production of Vitamin D in the human body as a result of inadequate exogenous intake over a long period of time undermine the most relevant risk factors to ascertain the difference of magnitude effect and compare each one. Sun exposure, including its determinants: time of day, application of sunscreen, sunbathing, nature of work and residence, outdoor/physical activity, and clothing as cultural behavior are significant in assessing the magnitude of risks posed on developing Vitamin D deficiency. Countries with latitudes near the equator, like the United Arab Emirates, receive more sunlight and therefore exposure influences the prevalence of Vitamin D deficiency and the cutaneous synthesis of vitamin $\mathrm{D}_{3}$. It's important to have "sun exposure between the hours of 10:00 and 15:00 in the spring, summer, and autumn, because this is the only time when enough UVB photons reach the earth's surface to produce vitamin $\mathrm{D}_{3}$ in the skin"13 The amount of sunbathing, in accordance with skin pigmentation, mainly affects the degree of being exposed to the sun where one practicing sunbathing will often result in achieving the recommended intake of Vitamin $\mathrm{D}$ as opposed to not sunbathing, however it is notable that lighter pigmented people absorb more than dark pigmented due to the latter requiring a larger amount of exposure to reach levels of the former ${ }^{\text {I4 }}$ Furthermore, sunscreens with an Sun protection factor factor of 8 reduce the skin's synthesis of vitamin $\mathrm{D}_{3}$ by $>95 \%$ whereas an Sun protection factor factor of 15 reduces synthesis by $>98 \%$. The frequency of sunscreen application is also of great significance in relation to causing Vitamin D deficiency, especially due to the possibility of women using it much more than men.

Cultural behavior, including clothing habits, outdoor activity(s) and the nature of work and educational environment, as well as the nutritional habits and diet further, influence the risk of developing Vitamin D deficiency, especially among women. Limiting outdoor and physical activities by being house-bound and leading a sedentary lifestyle can also have a large impact on Vitamin D deficiency. More importantly, urbanization is an important factor in the Middle East, due to most women and men working or studying almost all day indoors in buildings, institutions, and offices equipped with air conditioning. Their frequent use of enclosed transportation as opposed to walking outside also relates to the nature of their work, therefore causing decreased, or lack of, sun exposure over a long period of time.

A few food sources contain natural vitamin D including oily fish such as sardines, salmon, and mackerel, as well as some mushrooms are good sources of vitamin $\mathrm{D}_{3}$. Cod liver oil is considered to be an excellent source of vitamin $\mathrm{D}_{3}$; egg yolks are quite controversial "amounts are highly variable (usually no more than 50 IU per yolk), and the cholesterol content of egg yolks makes this a poor source of vitamin D"16 Finally, fortified foods, which have been previously discussed to have caused Vitamin D toxicity, are also an important source however are subject to availability in each country and to every individual depending on habit and economic status. Nevertheless, it represents a significant risk factor to consider, where orange juice, milk, yoghurts, and breakfast cereals are among the well-known products to be fortified with Vitamin $\mathrm{D}_{3}$. The table below derived from the USDA National Nutrient Database for Standard Reference clearly depicts the concentration of Vitamin D in natural and fortified foods.

The incidence of obesity and its related BMI indexes pertaining to the different categories of weight is a significant risk factor affecting the development of an increased risk of Vitamin D deficiency. According to Tsiaras W.G. and Weinstock M.A., 2010, vitamin D obtained from diet or fortified foods, as well as the cutaneous synthesis of Vitamin $\mathrm{D}_{3}$ is absorbed by adipose (fat) cells, and suspected to be stored for long periods of time in order to be used at times of necessary need, such as during the winter season ${ }^{17}$ Numerous studies have been conducted to assess the relationship between obesity and the risk of developing Vitamin D deficiency, where obese individuals tend to have lower serum concentrations of Vitamin $\mathrm{D}_{3}$ than others with normal body weights BMIs.

In one study, whole-body irradiation produced an increase in serum vitamin $\mathrm{D}_{3}$ levels in obese individuals [body mass index (BMI) $\left.30 \mathrm{~kg} / \mathrm{m}^{2}\right]$ that was $57 \%$ lower than that in age-matched, normal weight controls (BMI 25). In the same study, peak serum vitamin D2 concentrations after intake of an oral dose of 50,000 IU of vitamin D2 inversely correlated with BMI (54) ${ }^{18}$ Therefore, obesity causes decreased bioavailability of cutaneous and dietary Vitamin $\mathrm{D}$ due to obese individuals retaining their endogenous concentrations of Vitamin D and storing in adipose cells, thus not being metabolized or used by the body adequately. 


\section{CONCLUSION}

This study's aim is analyzing the magnitude effect of the examined risk factors relating to exogenous intake of Vitamin D and their effect on endogenous cutaneous synthesis of Vitamin $\mathrm{D}_{3}$, thus evaluating the factors contributing most to a higher risk of developing Vitamin $\mathrm{D}$ deficiency in the sample of 124 women studied. As clearly concluded from the statistical analysis and results obtained above, the cumulative effect of the following factors (Sunscreen type-Sun protection factor value, frequency of applying sunscreen, frequency and nature of sunbathing, and nature of the work/education environment) all contribute to a higher risk of developing Vitamin D deficiency in women in the UAE. The frequent use of a high Sun protection factor value, found to be 15 most frequently used by the sample of women in this study, in addition to rarely sunbathing and being in an indoor work/education environment daily all affect adequate sun exposure and therefore, intake of Vitamin D. Type of clothes (total or partial coverage) was not found to affect being exposed to the sun more than protection by frequent use of a high-value Sun protection factor or a daily indoor work/education environment imposing no sun exposure regardless of the type of clothes.

As for physical activity, weight, and regular consumption of foods with high contents of Vitamin D, more accurate and specific data needs to be collected in order to conclude their effects on sun exposure and risk of developing Vitamin $\mathrm{D}$ deficiency. However, whether being overweight/obese with increased storage of Vitamin $\mathrm{D}_{3}$ or inadequate intake of foods containing abundant amounts of Vitamin D cause higher risk of deficiency, the importance of living an active lifestyle with adequate sun exposure and regular consumption of healthy foods containing Vitamin D cumulatively prevent of development of deficiency in women.

\section{Highlights of Paper}

- Vitamin D deficiency is common among female women who live in the UAE.

- Around $65 \%$ of the participants have no exposure to sun light between 10 A.M to 3 PM.

- Food habits show a lack in vitamin D rich products to be included in the daily meals.

- This study recomends that women should practice some outdoor physical activety on regular bases.

\section{Author Profile}

- Dr. Muaed J. Alomar : Presently working as Assistant Professor in the clinical pharmacy department at Alain University, Alain, Abu Dhabi, UAE. Have been working as a lecturer, hospital pharmacy training coordinator and researcher for more than 13 years. Published many articles on clinical pharmacy practice, medication errors, DURs and adverse drug reactions. Previous experience in community pharmacy and as a product manager in the marketing department of MIDPHARMA pharmaaceutical industry, jordan. Good experience in the accreditation process and ACPE certification.

- Marie Christine: BSc. Pharmacy student.

\section{REFERENCES}

1. Holick MF, Michael F. Environmental Factors That Influence the Cutaneous Production of Vitamin D13. AMJ Clinical Nutrition. 1995; 61(3): 638S-45.

2. Holick MF, Michael F. Sunlight and Vitamin D for Bone Health and Prevention of Autoimmune Diseases, Cancers, and Cardiovascular Disease1,2,3,4. The American Journal of Clinical Nutrition. 2004; 80(6): 1678S-1688S.

3. 3., Teresa K, Groff, DO., Redmer J, Hounshell J, Schrager S, Vitamin D: An Evidence-Based Review. Journal of the Amercian Board of Family Medicine. 2009; 698-706 22(6): 698-706.

4. Mithal A, Wahl DA, Bonjour JP, Burckhardt P, Dawson-Hughes B, Eisman JA, El-Hajj Fuleihan G,. Josse RG. Global Vitamin D Status and Determinants of Hypovitaminosis D. Osteoporosis International. 2009; 20(11): 1807-820.

5. Saadi H, Dawodu A. Vitamin D deficiency in Arabian women and children: It is time for action. Emirates Med J. 2007; 25(3): 201-7.

6. Saadi HF, Nagelkerke N, Benedict S, Qazaq HS, Zilahi E, Mohamadiyeh MK. Predictors and relationships of serum 25 hydroxyvitamin $\mathrm{D}$ concentration with bone turnover markers, bone mineral density and vitamin $D$ receptor genotype in Emirati women. Bone. 2006; 39(5): 1136-43.
7. Sedrani SH, Elidrissy AW, El Arabi KM. Sunlight and vitamin D status in normal Saudi subjects. Am J Clin Nutr. 1983; 38(1): 181-5.

8. Zhen D, Liu L, Guan C, Zhao N, Tang T. High prevalence of vitamin $\mathrm{D}$ deficiency among middle-aged and elderly individuals in northwestern China: Its relationship to osteoporosis and lifestyle factors. Bone 2015; 71(1): 1-6.

9. Holick MF. Vitamin D and brain health: the need for vitamin D supplementation and sensible sun exposure. J Int Med. 2014; 277(1): 90-3.

10. Dursun E, Gezen-Ak D, Yilmazer S. A new mechanism for amyloid- $\beta$ induction of iNOS: vitamin D-VDR pathway disruption. J Alzheimers Dis. 2013; 36(1): 459-74.

11. Balden R, Selvamani A, Sohrabji F. Vitamin D deficiency exacerbates experimental stroke injury and dysregulates ischemia-induced inflammation in adult rats. Endocrinology 2012; 153(5): 2420-35.

12. Annweiler C, Dursun E, Féron F, Gezen-Ak D, et al. 'Vitamin D and cognition in older adults': updated international recommendations (Review). J Intern Med. 2014; 277(1): 45-57.

13. Wacker M, Holick MF. Vitamin D - effects on skeletal and extra skeletal health and the need for supplementation. Nutrients 2013; 5(1): 111-48. 
14. Hossein-nezhad A, Spira A, Holick MF. Influence of vitamin D status and vitamin D3 supplementation on genome wide expression of white blood cells: a randomized double-blind clinical trial. PLoS ONE. 2013; 8(3): e58725.

15. Gezen-Ak D, Dursun E, Yilmazer S. The effects of vitamin D receptor silencing on the expression of LVSCC-A1C and LVSCCA1D and the release of NGF in cortical neurons. PLoS ONE 2011; 6(3): e17553.

16. Ross AC, Manson JE, Abrams SA, et al. The 2011 report on dietary reference intakes for calcium and vitamin $D$ from the Institute of
Medicine: what clinicians need to know. J Clin Endocrinol Metab. 2011; 96(1): 53-8.

17. Simpson SJr, Blizzard L, Otahal P, Van der Mei I, Taylor B. Latitude is significantly associated with the prevalence of multiple sclerosis: a meta-analysis. J Neurol Neurosurg Psychiatry. 2011; 82(10): 1132-41.

18. U.S. Department of Agriculture, Agricultural Research Service. 2011. USDA National Nutrient Database for Standard Reference, Release 24. Nutrient Data Laboratory Home Page, http://www.ars. usda.gov/ba/bhnrc/ndl 